\title{
Consistency measure for the whole AHP decision making hierarchy
}

Masaaki Shinohara

Nihon University

Izumi-chou, Narashino

Chiba 275-8575, Japan

M7sinoha@cit.nihon-u.ac.jp
Keikichi Osawa

Nihon University

Izumi-chou, Narashino

Chiba 275-8575, Japan

k7oosawa@cit.nihon-u.ac.jp

Keywords: consistency index, CI, whole AHP decision making hierarchy, square root formula Summary: A new consistency measure for the whole AHP decision making hierarchy, $C_{A H P}$, is proposed, while conventional Saaty's consistency measure, $C_{\text {local }}$, gives information about logical consistency for a pairwise comparison matrix at a local judgment, such as at the goal node comparing pairs of criteria or at a criterion node comparing pairs of alternatives. First, conventional consistency measure, $C_{\text {local }}$ is shown to be proportional to certain discrepancy measure squared. Then, using this relationship, a formula is established, which relates a set of local consistency measures in an AHP hierarchy and the consistency measure for the whole AHP decision making hierarchy. The formula is then applied to decision making of university department selection, and it is shown that local consistency measures do not linearly affect the consistency measure for the whole AHP decision making. It is also mentioned that the proposed formula for $C I_{A H P}$ is a special case of the formula for $C I_{A N P}$, a consistency measure for the whole ANP decision making network.

\section{Introduction}

The consistency index CI, defined by $\left(\lambda_{\max }-n\right) /(n-1)$, gives information about logical consistency among pairwise comparison judgments in a perfect pairwise comparison case. When $\mathrm{CI}=0.0$, there is no logical inconsistency among the pairwise comparison judgments, or the judgment is considered $100 \%$ consistent. As the value of CI grows, the degree of logical inconsistency among the pairwise comparison judgments is also considered to grow. There have been discussions about what threshold value should be chosen for the value of CI beyond which the judgment is considered unacceptable.

In this paper, the concept of consistency measure for the whole AHP decision making hierarchy is proposed and an approach of integrating local consistency indexes $\mathrm{CI}_{\text {local }} \mathrm{S}$ into the global consistency index $\mathrm{CI}_{\mathrm{AHP}}$ is presented. Here, a local consistency index $\mathrm{CI}_{\text {local }}$ means a conventional $\mathrm{CI}$ which is for a local decision making node in an AHP decision making hierarchy, such as the goal node comparing pairs of criteria or a criterion node comparing pairs of alternatives, and the global consistency index $\mathrm{CI}_{\mathrm{AHP}}$ means proposed consistency measure for the whole AHP decision making hierarchy. Weighted square root formula for $\mathrm{CI}_{\mathrm{AHP}}$ is proposed in Chapter 2, reasons for the weighted square 
root formula are presented in Chapter 3, and the square root formula is applied to an actual AHP decision making problem in Chapter 4.

\section{Formula for $\mathbf{C I}_{\mathrm{AHP}}$}

Consider a three-level AHP diagram shown in Fig.1, where there are $M$ criteria and $N$ alternatives. At the goal node and at each criterion node, local consistency indexes are defined as below.

$\mathrm{CI}_{0}$ : consistency index for pairwise comparison judgments among criteria from the goal.

$\mathrm{CI}_{\mathrm{k}}$ : consistency index for pairwise comparison judgments among alternatives from criterion $k(k=1, \ldots, M)$.

Each of these CIs gives information about the degree of logical consistency at a local node in an AHP decision making hierarchy. Noting that a three-level AHP(Fig.1) can be equivalently viewed as a two-level AHP shown in Fig.2, there can exist a consistency measure for this two-level AHP, which is the consistency measure for the whole decision making hierarchy and is denoted by $\mathrm{CI}_{\mathrm{AHP}}$.

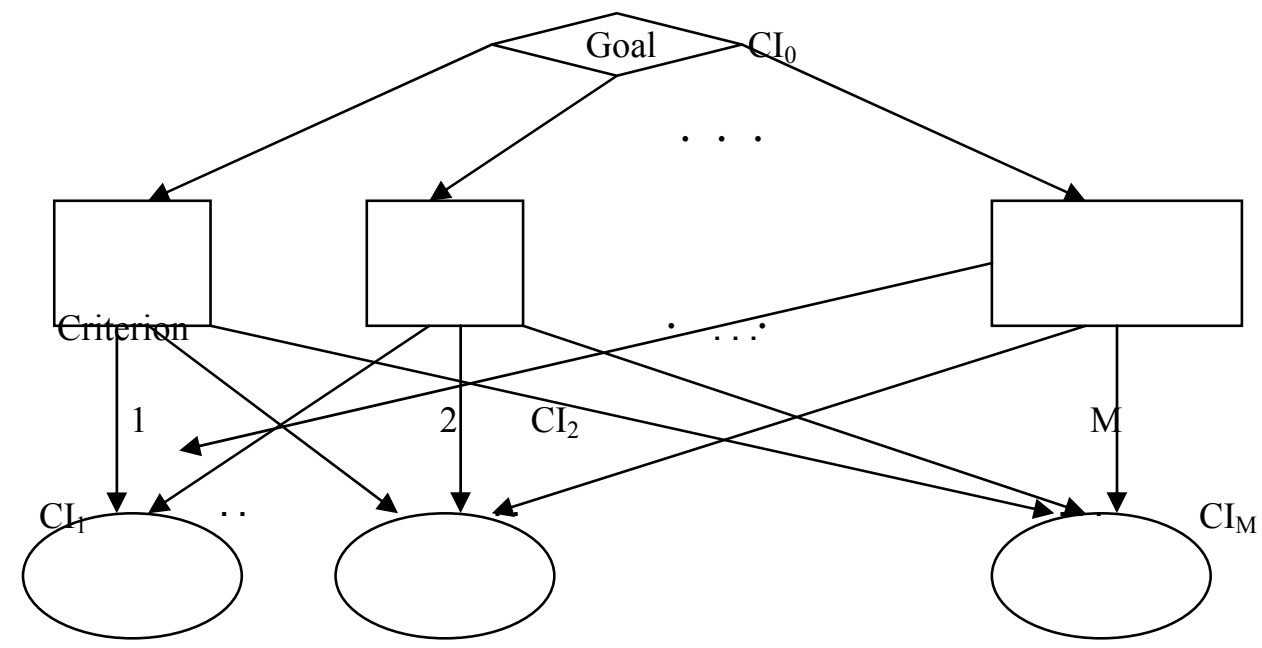

Alternative

Fig. 1 Three-level AHP diagram

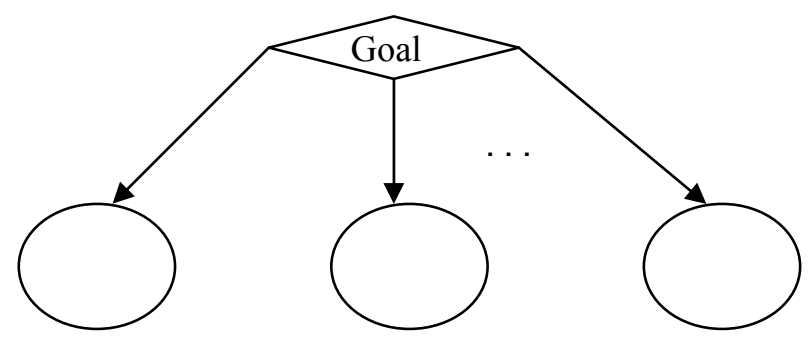


Alternative

1

$2 \cdot \ldots \cdot \mathrm{N}$

Fig. 2 Equivalent two-level AHP diagram

Next, we will present a formula of expressing $\mathrm{CI}_{\mathrm{AHP}}$ using $C I_{k}{ }^{\prime}(k=0,1,2, \ldots, M)$.

\section{Theorem 1}

In case of a three-level AHP shown in Fig.1, the consistency index for the whole decision making hierarchy, $\mathrm{CI}_{\mathrm{AHP}}$, is approximately given by (1) or (2), where $w_{k}$ is estimated priority weight for criterion $k$ and $\sum w_{k=1}$.

$$
\begin{aligned}
& C I_{A H P} \fallingdotseq\left(\sqrt{C I_{0}}+\sum_{k=1}^{M} w_{k} \sqrt{C I_{k}}\right)^{2} \\
& \sqrt{C I_{A H P}} \fallingdotseq \sqrt{C I_{0}}+\sum_{k=1}^{M} w_{k} \sqrt{C I_{k}}
\end{aligned}
$$

Note that square root of $\mathrm{CI}_{\mathrm{AHP}}, \sqrt{C I_{A H P}}$, is expressed as weighted sum of square roots of local consistency indexes, $\sqrt{C I_{k}}(k=0,1,2, \ldots, M)$, instead that $\mathrm{CI}_{\mathrm{AHP}}$ is expressed as weighted linear sum of local consistency indexes, $C I_{k} s(k=0,1,2, \ldots, M)$.

Although they may not give an exact proof for Theorem 1, in the next section we will show two reasons why the square root of $\mathrm{CI}_{\mathrm{AHP}}$ is given by weighted sum of square roots of local consistency indexes $C I_{k} s(k=0,1,2, \ldots, M)$.

\section{3 . Two reasons for the square root formula}

Two reasons (or two pieces of evidence) for the square root formula (1) or (2) are shown in this chapter.

The first piece of evidence(Reason I) is based on the fact about the meaning of CI that a local 
consistency index $\mathrm{CI}$ is approximated to $\delta^{2} / 2$, or $2 C I \doteqdot \delta^{2}$, where $\delta$ can be interpreted as a certain kind of deviation of pairwise comparison measurement error $\left\{e_{i j}\right\}$ from 1.0 ( detailed notations will be explained later).

The second piece of evidence(Reason II) is based on an analytical fact that under certain restricted conditions the square root of global consistency measure is analytically approximated to weighted sum of square roots of local consistency measures.

\subsection{Reason I from formula $\delta=\sqrt{2 C I}$}

Let $\mathrm{a}_{\mathrm{ij}}$ be (i,j)-pairwise comparison measurement value and $\mathrm{w}_{\mathrm{ij}}=\mathrm{w}_{\mathrm{i}} / \mathrm{w}_{\mathrm{j}}$ be (i,j)-pairwise comparison estimate, where $\mathrm{w}_{\mathrm{k}}$ is estimated priority weight for item $\mathrm{k}$.

Then, (i,j)-pairwise comparison measurement error $\mathrm{e}_{\mathrm{ij}}$ is defined by Eq.(3)or Eq.(4).

$$
\begin{aligned}
a_{i j}=w_{i j} \cdot e_{i j} \\
e_{i j}=a_{i j} / w_{i j}
\end{aligned}
$$

If we assume the positive reciprocality of pairwise comparison measurement matrix $A=\left\{a_{i j}\right\}$, its associated pairwise comparison measurement error matrix $\mathrm{E}=\left\{\mathrm{e}_{\mathrm{ij}}\right\}$ is also positive-reciprocal.

Let $\overline{\mathrm{e}}$ be expectation of $\mathrm{e}_{\mathrm{ij}}$ over $(\mathrm{i}, \mathrm{j})$ pairs $(\mathrm{i} \neq \mathrm{j})$, and be denoted by $\operatorname{Exp}\left(\mathrm{e}_{\mathrm{ij}}\right)$, where Exp stands for 'expectation'.

$$
\begin{aligned}
\bar{e} & =\operatorname{Exp}\left(e_{i j}\right) \\
& =\frac{1}{n(n-1)} \sum_{i \neq j} e_{i j}
\end{aligned}
$$

Let $\mathrm{e}_{\mathrm{ij}}=1+\delta_{\mathrm{ij}}\left(\delta_{\mathrm{ij}}>-1\right)$, then approximately speaking, following approximation (6) holds.

$$
\begin{aligned}
1+\delta_{i j}+\left(1+\delta_{i j}\right)^{-1} \doteqdot & + \\
& +\delta_{i j}+1-\delta_{i j}+\delta_{i j}^{2} \\
& =2+\delta_{i j}^{2}
\end{aligned}
$$

Using this approximation (6), $\operatorname{Exp}\left(\mathrm{e}_{\mathrm{ij}}\right)$ is approximately expressed in another way as by (7).

$$
\operatorname{Exp}\left(e_{i j}\right) \doteqdot 1+\frac{1}{2} \bar{\delta}^{2}
$$




$$
\bar{\delta}^{2}=\frac{2}{n(n-1)} \sum_{i>j} \delta_{i j}^{2}
$$

It is well-known that the value $\overline{\mathrm{e}}=\operatorname{Exp}\left(\mathrm{e}_{\mathrm{ij}}\right)$ is related to $\mathrm{CI}$ for $\mathrm{A}=\left\{\mathrm{a}_{\mathrm{ij}}\right\}$ by Eq.(9).

$$
C I=\operatorname{Exp}\left(e_{i j}\right)-1
$$

Combining Approximation (7) and Eq.(9), following formula (10) relating CI to $\bar{\delta}^{2}$ is obtained.

$$
2 C I \doteqdot \bar{\delta}^{2}
$$

Here, $\overline{\delta^{2}}$ is the expectation of $\delta_{i j}$ squared over $(i, j)$ pairs with $i>j$.

By renumbering i's and j's, we can choose set of $(i, j)$ pairs with $i>j$ so that

$e_{i j} \geqq 1$ holds for the set of renumbered $\left(i^{*}, j^{*}\right)$ pairs with $i^{*}>j^{*}$.

In this case, Eq.(8) can be very roughly approximated to Eq.(11).

$$
\begin{aligned}
& \overline{\delta^{2}} \fallingdotseq \bar{\delta}^{2} \\
& \bar{\delta}=\frac{2}{n(n-2)} \sum_{i^{*}>j^{*}} \delta_{i^{*} j^{*}}
\end{aligned}
$$

Finally, approximation (13) is obtained.

$$
2 \mathrm{CI} \fallingdotseq \bar{\delta}^{2}
$$

Here, $\bar{\delta}$ (or simply it is denoted by $\delta$ hereafter) is mean discrepancy (or deviation) from one in measurement data error of $a_{i j}$ or $a_{j i}$ with its value greater than or equal to one.

Approximation (11) should be $\overline{\delta^{2}} \geqq \bar{\delta}^{2}$ mathematically and the approximation (11) may be valid only under certain conditions. But a lot of simulation runs and experiment runs were carried out and it has been confirmed that approximation (13) is valid under various conditions (see [3]).

Formula (13) is rewritten as in (14).

$$
\delta=\sqrt{2 C I}
$$

This means that the mean discrepancy $\delta$ in a pairwise comparison measurement is approximately equal to the square root of two times the consistency index CI for the set of pairwise comparison judgments. Note that any local priority weight also has this 'mean discrepancy tendency'. Then, it is 
expected that following relationship (15) or (16) holds among $\Delta$ and $\delta_{k} s(k=0,1,2, \ldots, M)$.

$$
\begin{aligned}
& (1+\Delta)=\left(1+\delta_{0}\right)\left(1+\sum_{\mathrm{k}=1}^{\mathrm{M}} \mathrm{w}_{\mathrm{k}} \delta_{\mathrm{k}}\right) \\
& \Delta \doteqdot \bar{\delta}_{0}+\sum_{\mathrm{k}=1}^{\mathrm{M}} \mathrm{w}_{\mathrm{k}} \delta_{\mathrm{k}}
\end{aligned}
$$

$\Delta \quad$ : mean discrepancy for the whole AHP decision making hierarchy of Fig.1.

$\delta_{0}$ : mean discrepancy for pairwise comparison judgments among criteria from the goal.

$\delta_{\mathrm{k}}$ : mean discrepancy for pairwise comparison judgments among alternatives from criterion $\mathrm{k}(\mathrm{k}=1, \ldots, \mathrm{M})$.

We also expect followings hold.

$$
\begin{aligned}
\Delta & \fallingdotseq \sqrt{2 C I_{A H P}} \\
\delta_{\mathrm{k}} & \fallingdotseq \sqrt{2 C I_{k}} \quad(k=0,1,2, \ldots, M)
\end{aligned}
$$

Substituting (17) and (18) into (16), we obtain following weighted square root formula (19).

$$
\sqrt{C I_{A H P}} \fallingdotseq \sqrt{C I_{0}}+\sum_{k=1}^{M} w_{k} \sqrt{C I_{k}}
$$

\subsection{Reasonll from analytical result}

In this section we briefly review the analytical result which is obtained in [4], where we directly tried to derive the consistency index for the 2-level AHP diagram shown in Fig.2 under certain restricted conditions.

First, following notations are defined.

$A(0)=\left\{a_{i j}(0)\right\}: M \times M$ pairwise comparison matrix among $M$ criteria from the viewpoint of the goal. $A(k)=\left\{a_{i j}(k)\right\}: N \times N$ pairwise comparison matrix among $N$ alternatives from the viewpoint of criterion $k$.

$P=\left\{p_{i j}\right\}: N \times N$ pairwise comparison matrix among $N$ alternatives directly from the viewpoint of the goal in the 2-level AHP shown in Fig.2.

$\mathrm{CI}_{2 \text {-level }}$ : Consistency index for $P=\left\{p_{i j}\right\}$.

We do not treat a general case but a limited case of AHP where the number of alternatives $N=3$ and the pairwise comparison matrix $\mathrm{P}$ for the equivalent 2-level AHP is composed from each of pairwise 
comparison matrixes $A(k)$ 's $(k=1,2, \ldots, M)$ by their elementwise geometric mean (20).

$$
p_{i j}=\prod_{k=1}^{M} a_{i j}(k)^{W k}
$$

Then, we get following Theorem 2 and Theorem 3.

\section{Theorem 2}

If all pairwise comparison matrixes $A(k) \mathfrak{s}(k=1,2, \ldots, M)$ are positive-reciprocal, then $\mathrm{P}=\left\{\mathrm{p}_{\mathrm{ij}}\right\}$ defined by (20) is also positive-reciprocal.

\section{Theorem 3}

If all pairwise comparison matrixes $A(k) s(k=1,2, \ldots, M)$ are positive-reciprocal and $\mathrm{P}=\left\{\mathrm{p}_{\mathrm{ij}}\right\}$ is defined by geometric composition of $A(k) s(k=1,2, \ldots, M)$, then following relationship (21) or (22) holds for $N=3$.

$$
\begin{aligned}
& \sqrt{C I_{2-\text { level }}} \fallingdotseq\left(\sum_{k=1}^{M} w_{k} \sqrt{C I_{k}}\right) \\
& C I_{2-\text { level }} \fallingdotseq\left(\sum_{k=1}^{M} w_{k} \sqrt{C I_{k}}\right)^{2}
\end{aligned}
$$

As indicated by formula (21), the square root of the consistency index for the whole 2-level AHP shown in Fig.2 is approximated to weighted sum of square roots of local consistency indexes.

Although this analytical result is obtained under certain restricted conditions such as transformation of 3-level AHP into 2-level AHP, geometric mean composition of comparison matrixes, and the number of alternatives $N=3$, and the effect of $\mathrm{Cl}_{0}$ (the consistency index for pairwise comparison judgments among criteria from the goal) does not appear explicitly in the right hand side of formula (21) or (22), this result suggests the validity of the weighted square root formula of Theorem 1. If we add the term $\sqrt{C I_{0}}$ in the right hand side of (21), the formula completely coincides with the formula of Theorem 1 .

\section{Application of the formula to department selection decision making}

Our College of Industrial Technology, Nihon University, consists of seven engineering departments as of April 1, 2007. They are mechanical (ME), electrical(EL), civil(CV), architectural(AR), chemical(CH), management(MA), and mathematical information(MI). We conducted a questionnaire survey to 30 students who belong to the mathematical information department and asked them in choosing department which criterion, they think, is important and which department (alternative), they think, is effective from the viewpoint of each criterion. All the questions asked are in the form of pairwise comparison. Fig.3 shows the AHP diagram for this 
questionnaire. The goal is 'which department to chose', and four criteria are considered. They are university life environment(LE), field of interest(FI), occupational opportunity(OO), and brand image(BI).

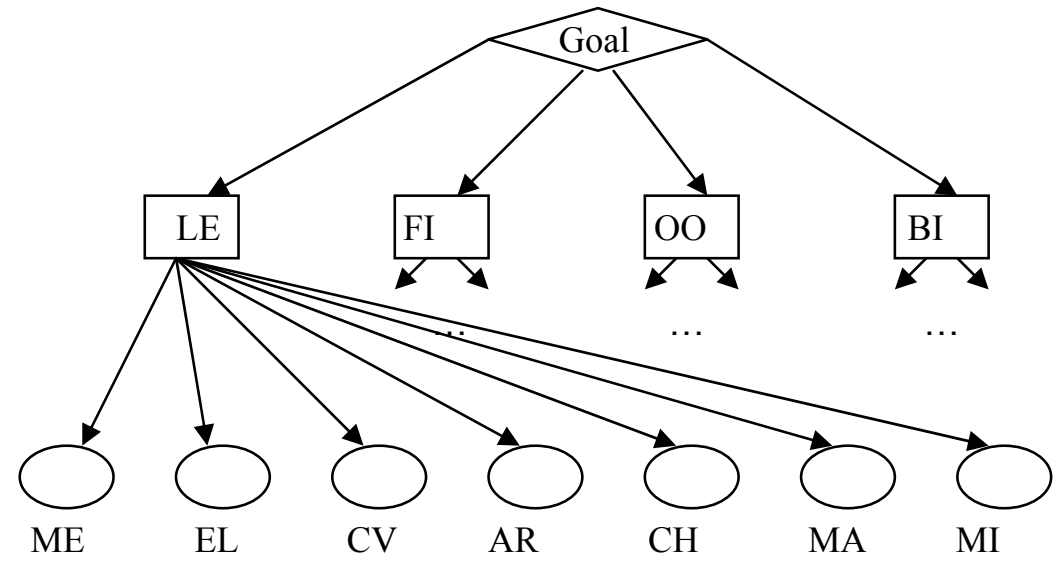

\section{Fig.3 AHP diagram for department selection}

Two types of consistency measure for the whole AHP decision making hierarchy, traditional weighted arithmetic mean formula $\mathrm{CI}_{\mathrm{AHPtraditional}}$ and proposed weighted square root mean formula $\mathrm{CI}_{\mathrm{AHP} \text { proposal }}$ are calculated, and followings are observed.

$$
\begin{gathered}
\mathrm{CI}_{\mathrm{AHPtraditional}}=C I_{0}+\sum_{k=1}^{M} w_{k} C I_{k} \\
\mathrm{CI}_{\text {AHPproposal }}=\left(\sqrt{C I_{0}}+\sum_{k=1}^{M} w_{k} \sqrt{C I_{k}}\right)^{2}
\end{gathered}
$$

Observation1: When $\mathrm{CI}_{0} \neq 0$, there is a general tendency ' $\mathrm{CI}_{\mathrm{AHPtraditional}} \leqq \mathrm{CI}_{\mathrm{AHPproposal}}$ ', and the difference ' $\mathrm{CI}_{\mathrm{AHPproposal}}-\mathrm{CI}_{\mathrm{AHPtraditional}}$ ' tends to grow with values of CIs.

Observation2: When $\mathrm{CI}_{0}=0$, there is a general tendency ' $\mathrm{CI}_{\mathrm{AHPtraditional}} \geqq \mathrm{CI}_{\mathrm{AHPproposal}}$ ', which can be proved, and the difference ' $\mathrm{CI} \mathrm{I}_{\mathrm{AHPtraditional}}-\mathrm{CI}_{\mathrm{AHPproposal}}$ ' is small, or $\mathrm{CI}_{\mathrm{AHPtraditional}} \fallingdotseq \mathrm{CI}_{\mathrm{AHPproposal}}$.

Observation3: Each portion of $C I_{0}$ or $C I_{k}(k=0,1,2, \ldots, M)$ in $\mathrm{CI}_{\mathrm{AHPtraditional}}$ is not always close to that of $\sqrt{C I_{0}}$ or $\sqrt{C I_{k}}(k=0,1,2, \ldots, M)$ in $\sqrt{\mathrm{CI}_{\mathrm{AHPproposal}}}$. See next example data of Student No.5, where the biggest portion in $\mathrm{CI}_{\mathrm{AHPtraditional}}$ is attained by $w_{2} \mathrm{CI}{ }_{2}$, while the biggest portion 
in $\sqrt{\mathrm{CI}_{\mathrm{AHPproposa}}}$ is attained by $\sqrt{C I_{0}}$.

\section{[Example data of Student No.5]}

\begin{tabular}{|r|r|r|r|}
\hline \multicolumn{4}{|c|}{ Amone Criteria from Gosl } \\
\hline 1 & 05 & 1 & 2 \\
\hline 2 & 1 & 2 & 3 \\
\hline 1 & 05 & 1 & 3 \\
\hline 05 & 0.333 & 0.333 & 1 \\
\hline
\end{tabular}

\begin{tabular}{|c|c|c|c|c|c|c|c|c|c|c|c|c|c|}
\hline \multicolumn{7}{|c|}{ Amone Alternatives from Criterion 1} & \multicolumn{7}{|c|}{ Among Alternatives from Criterion 2} \\
\hline 1 & 1 & 2 & 05 & 05 & 0333 & 1 & 1 & 1 & 2 & 1 & 05 & 1 & 05 \\
\hline 1 & 1 & 3 & 0333 & 1 & 0.333 & 2 & 1 & 1 & 05 & 1 & 0.5 & 2 & 0.5 \\
\hline 0.5 & 0333 & 1 & 025 & 0.5 & 0.25 & 05 & 0.5 & 2 & 1 & 0.333 & 0.333 & 1 & 0.333 \\
\hline 2 & 3 & 4 & 1 & 2 & 3 & 3 & 1 & 1 & 3 & 1 & 1 & 1 & 0.5 \\
\hline 2 & 1 & 2 & 0.5 & 1 & 0.5 & 0.5 & 2 & 2 & 3 & 1 & 1 & 2 & 0.333 \\
\hline 3 & 3 & 4 & 0333 & 2 & 1 & 4 & 1 & 0.5 & 1 & 1 & 0.5 & 1 & 0333 \\
\hline 1 & 0.5 & 2 & 0.333 & 2 & 025 & 1 & 2 & 2 & 3 & 2 & 3 & 3 & \\
\hline
\end{tabular}

\begin{tabular}{|l|l|}
\hline Among Alternatives from Criterion 3 & Among Alternatives from Criterion 4 \\
\hline
\end{tabular}

\begin{tabular}{|r|r|r|r|r|r|r|r|r|r|r|r|r|r|r|}
\hline 1 & 1 & 3 & 2 & 2 & 2 & 0.333 & 1 & 1 & 3 & 1 & 0.333 & 0.5 & 0.5 \\
\hline 1 & 1 & 2 & 1 & 2 & 2 & 0.5 & 1 & 1 & 3 & 1 & 0.5 & 05 & 0.5 \\
\hline 0.333 & 0.5 & 1 & 0.333 & 0.333 & 0.5 & 0.25 & 0.333 & 0.333 & 1 & 0.5 & 0.333 & 0.25 & 0.333 \\
\hline 0.5 & 1 & 3 & 1 & 1 & 2 & 0.333 & 1 & 1 & 2 & 1 & 0.5 & 0.5 & 05 \\
\hline 0.5 & 0.5 & 3 & 1 & 1 & 2 & 0.333 & 3 & 2 & 3 & 2 & 1 & 1 & 0.5 \\
\hline 0.5 & 0.5 & 2 & 0.5 & 0.5 & 1 & 0.333 & 2 & 2 & 4 & 2 & 1 & 1 & 1 \\
\hline 3 & 2 & 4 & 3 & 3 & 3 & 1 & 2 & 2 & 3 & 2 & 2 & 1 & 1 \\
\hline
\end{tabular}

$\mathrm{w}_{1}=0.222, \mathrm{w}_{2}=0.418, \mathrm{w}_{3}=0.250, \mathrm{w}_{4}=0.110, \mathrm{CI}_{0}=0.0152, \mathrm{CI}_{1}=0.0754, \mathrm{CI}_{2}=0.0706, \mathrm{CI}_{3}=0.0368$, $\mathrm{CI}_{4}=0.0701, \mathrm{CI}_{\mathrm{AHPtraditional}}=0.0785, \mathrm{CI}_{\mathrm{AHPproposal}}=0.1389$.

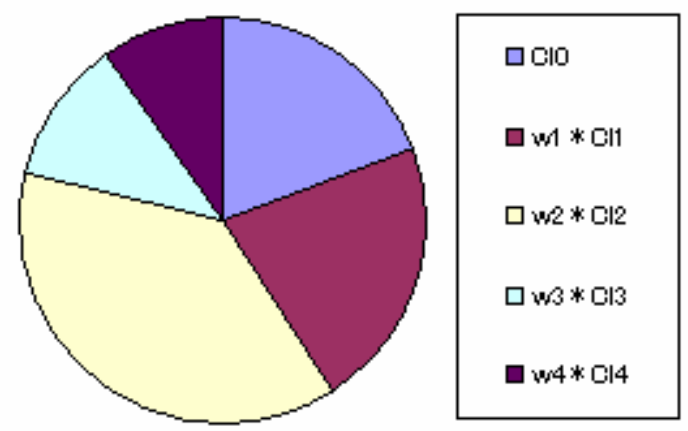

Fig.4 Portions of weighted CIs in CIAHPtraditional

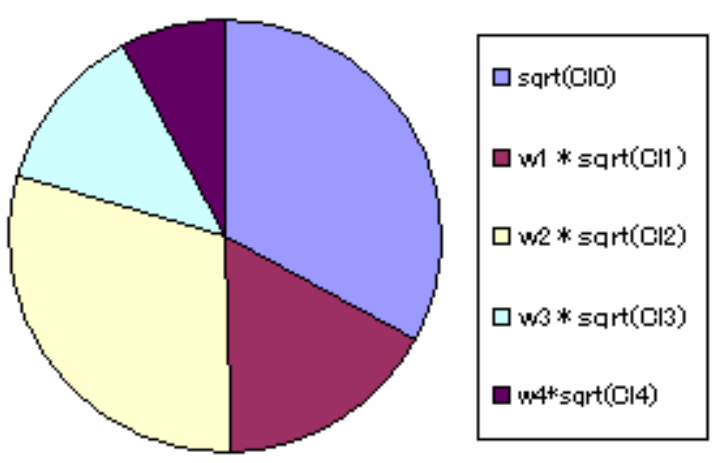


Fig.5 Portions of weighted square rooted CIs in $\sqrt{\mathrm{CI}_{\text {AHPproposal }}}$

\section{Conclusion}

A new consistency measure for the whole AHP decision making hierarchy is proposed and proposed square root formula is numerically compared with traditional arithmetic mean formula through a decision making problem of department selection. Note that proposed square root formula for AHP is a special version of the square root formula for ANP [5], shown by (25).

$$
C I_{A N P} \approx\left(\sum x_{i} \sqrt{C I_{i}}\right)^{2}
$$

Measuring directly virtual pairwise comparison matrix $\mathrm{P}$ for the equivalent 2-level AHP and checking the validity of proposed square root formula is a future research subject.

\section{References}

[1] T.L.Saaty: The Analytic Hierarchy Process (Mc-Graw Hill)(1980).

[2] T.L.Saaty: The Analytic Network Process (RWS-Publication)(1996).

[3] Kazuya Inamine, Itaru Goto, Masaaki Shinohara and Keikichi Osawa: Relationship between consistency index CI and multiplicative error discrepancyס, Proceedings of the 2005 Fall National Conference of Operations Research Society of Japan (in Japanese), pp.148-149 (2005).

[4] Masaaki Shinohara Keikichi Osawa and Ken Shinohara: Local consistency vs global consistency in AHP, Proceedings of the 2006 Fall National Conference of Operations Research Society of Japan (in Japanese), pp.242-243 (2006).

[5] Masayuki Utugizaki, Miki Udagawa, Masaaki Shinohara and Keikichi Osawa: Consistency index for the whole decision making, Proceedings of DEA Symposium 2007, Osaka University (in Japanese), pp.102-105 (2007). 